\title{
Evaluation of Insecticide Mixtures against Larval Population of Spotted Pod Borer, Maruca vitrata in Cowpea
}

\author{
Banka Kanda Kishore Reddy* and Jangam Hampaiah \\ Department of Entomology, Kerala Agricultural University, College of Agriculture, \\ Vellayani, Thiruvananthapuram, Kerala, India \\ *Corresponding author
}

\section{A B S T R A C T}

Keywords

Efficacy, Insecticide mixtures, Pod borer.

Article Info

Accepted:

15 June 2018

Available Online:

10 July 2018

\begin{abstract}
Evaluation of efficacy of insecticide mixtures against the spottedpod borer, Maruca vitrata was conducted at College of Agriculture, Vellayani, Thiruvananthapuram during 2017. The result revealed that no larva was found in the treatment lambda cyhalothrin $4.6 \%+$ chlorantraniliprole $9.3 \%$ ZC @ $0.50 \mathrm{~mL} \mathrm{~L}^{-1}$ and chlorantraniliprole $8.8 \%+$ thiamethoxam $17.5 \% \mathrm{SC} @ 0.30 \mathrm{~mL} \mathrm{~L}^{-1}$ treated plants after 5 days of spraying. Then from 7 seven days after spraying no larvae was recorded in the treatment lambda cyhalothrin $4.6 \%+$ chlorantraniliprole $9.3 \% \mathrm{ZC} @ 0.50 \mathrm{~mL} \mathrm{~L}^{-1}$ only and less number of larvae was recorded in chlorantraniliprole $8.8 \%$ + thiamethoxam $17.5 \% \mathrm{SC} @ 0.30 \mathrm{~mL} \mathrm{~L}^{-1}$ followed by beta cyfluthrin $8.91 \%$ + imidacloprid $19.81 \%$ SC @ $0.40 \mathrm{~mL} \mathrm{~L}^{-1}$. The existing management practices with single insecticides are meagre to meet the demand. Hence the present findings of the experiment concluded that the use of ready-mix formulation lambda cyhalothrin $4.6 \%$ + chlorantraniliprole $9.3 \% \mathrm{ZC} @ 0.50 \mathrm{~mL} \mathrm{~L}^{-1}$ is found to be superior to control larval population in cowpea.
\end{abstract}

\section{Introduction}

Cowpea (Vigna unguiculata subsp. Sequipedalis (L.) Verdc.) generally termed as yard long bean is the most widely adapted, versatile and nutritious grain legume crop in tropical and sub- tropical countries. As many as 21 insect pests of different groups are recorded damaging the cowpea crop from germination to maturity (Sardhana and Verma, 1986). Spotted pod borer is the most dangerous and potential pest creating considerable damage to the crops by infesting flowers and pods.
Webbing of leaves and scrapping followed by feeding on developing seeds inside the pods results in higher yield loss up to 60 per cent in cowpea (Pandey et al., 1991). It is reported that the loss due to pod damage alone goes 42 to 80 per cent (Halder and Srinivasan, 2011). M.vitrata larvae feed on flowers, buds and pods by webbing them. This typical feeding protects the larvae from natural enemies and other adverse factors, including insecticides. Moths prefer to oviposit at the flower bud stage. Third to fifth instar larvae are capable of boring into the pods and occasionally into 
peduncle and stems (Vijayasree, 2013). However, widespread and long-term use of single insecticides resulted in insecticide resistance and biomagnification of insecticides and forcing the farmers to use higher dose and more application frequency by the way chemical; cost labour cost will be increased. Pesticide mixtures may enhance the suppression of arthropod pest population due to either synergistic interaction or potentiation between or among pesticides that are mixed together. It has been proposed that pesticide mixtures may delay the onset of resistance developing in arthropod pest populations (Skylakakis, 1981; Mani, 1985; Mallet, 1989). Mixtures of insecticides provide technical advantages for controlling pests in a broad range of settings, typically by increasing the level of target pest control and/or broadening the range of pests controlled (IRAC, 2018). Recent reports revealed that the pest has developed resistance to the conventional insecticides which are repetitively using from long times. Moreover, no studies have been carried out in Kerala on the efficacy of insecticide mixtures against pests of cowpea.

\section{Materials and Methods}

The following insecticide mixtures will be tested for their efficacy against pod borers M.vitrata and the insecticides will be applied at 5-10\% infestation level.

$\begin{array}{ll}\text { Design } & : \text { RBD } \\ \text { Replications } & : 3 \\ \text { Treatments } & : 9 \\ \text { Insecticide } & \text { mixtures } \quad \text { (Ready-mix }\end{array}$

formulations) were used in the study along with two standard checks viz., chlorantraniliprole, thiamethoxam and one treatment as manual hand mixing of chlorantraniliprole and thiamethoxam (1:1) @ $0.3 \mathrm{~mL} \mathrm{~L}^{-1}$.
Fifteen plants were selected randomly and number of larvae present in flowers of each plant were counted after $1,3,5,7,10$ and 15 days after spraying.

\section{Statistical analysis}

The data collected were subjected to analysis of variance (ANOVA) after applying appropriate transformations.

\section{Results and Discussion}

\section{Larval population}

The lowest number of larvae was found in plants treated with hand mixed product of chlorantraniliprole $18.5 \% \mathrm{SC}+$ thiamethoxam $25 \%$ WG (1:1)@0.3 mL L ${ }^{-1}$ (1.00) after first day of spraying and it was significantly different from other treatments. Larval population was found in plants treated with chlorantraniliprole $8.8 \%+$ thiamethoxam $17.5 \%$ SC @ $0.3 \mathrm{~mL} \mathrm{~L}^{-1}$, lambda cyhalothrin $4.6 \%$ + chlorantraniliprole $9.3 \%$ ZC @ 0.5 $\mathrm{mL} \mathrm{L}^{-1}$, chlorantraniliprole18.5\% SC @ 0.3 $\mathrm{mL} \mathrm{L}^{-1}$ were 2.00 each. Higher population of larvae was recorded in thiamethoxam $12.6 \%$ + lambda cyhalothrin $9.5 \% \mathrm{ZC} @ 0.3 \mathrm{~mL} \mathrm{~L}^{-1}$ (3.67), thiamethoxam $25 \%$ WG @ $0.3 \mathrm{~mL} \mathrm{~L}^{-1}$ (3.67) followed by flubendiamide19.92\%+ thiacloprid $19.92 \%$ SC @ $0.5 \mathrm{~mL} \mathrm{~L}^{-1}$ (3.33) and they were significantly different as compared to control (5.67).

Infestation was reduced after three days of treatment and lower number of larvae was observed in lambda cyhalothrin $4.6 \%+$ chlorantraniliprole $9.3 \%$ ZC @ $0.5 \mathrm{~mL} \mathrm{~L}^{-1}$ (0.33) and it was statistically on par with plants treated with hand mixed product of chlorantraniliprole $18.5 \% \mathrm{SC}+$ thiamethoxam $25 \%$ WG @ (1:1) (0.67), chlorantraniliprole18.5\% SC @ $0.3 \mathrm{~mL} \mathrm{~L}^{-1}$ (1.00), chlorantraniliprole $8.8 \%+$ thiamethoxam 17.5\% SC @ $0.3 \mathrm{~mL} \mathrm{~L}^{-1}$ (1.00). Similarly, number of larvae found in 
thiamethoxam 25\% WG @ $0.3 \mathrm{~mL} \mathrm{~L}^{-1}$, thiamethoxam $12.6 \%$ + lambda cyhalothrin $9.5 \%$ ZC @ $0.3 \mathrm{~mL} \mathrm{~L}^{-1}$, beta cyfluthrin 8.49 $\%+$ imidacloprid $19.81 \%$ SC @ $0.4 \mathrm{~mL} \mathrm{~L}^{-1}$ were $2.67,2.67,2.00$ respectively and they were significantly different when compared to untreated control (6.33).

After five days of spraying no larvae was found in chlorantraniliprole $8.8 \%+$ thiamethoxam $17.5 \%$ SC @ $0.3 \mathrm{~mL} \mathrm{~L}^{-1}$, lambda cyhalothrin $4.6 \quad \% \quad+$ chlorantraniliprole $9.3 \%$ ZC @ $0.5 \mathrm{~mL} \mathrm{~L}^{-1}$ followed by flubendiamide19.92\% +thiacloprid $19.92 \%$ SC @ $0.5 \mathrm{~mL} \mathrm{~L}^{-1}$ (0.67), chlorantraniliprole18.5\% SC @ $0.3 \mathrm{~mL} \mathrm{~L}^{-1}$ (0.67), hand mixed product of chlorantraniliprole $18.5 \% \mathrm{SC}+$ thiamethoxam $25 \%$ WG @ (1:1) (1.33). While, the treatment thiamethoxam 25\%WG@0.3 mL L L $^{-1}$ shown a population of 3.33 and it was statistically on par with thiamethoxam $12.6 \%$ + lambda cyhalothrin 9.5\% ZC @ $0.3 \mathrm{~mL} \mathrm{~L}^{-1}$ (2.67) followed by beta cyfluthrin $8.49 \%+$ imidacloprid $19.81 \%$ SC $@ 0.4$ mL L-1 (2.00).

No larva was recorded from plants treated with lambda cyhalothrin $4.6 \%+$ chlorantraniliprole $9.3 \% \mathrm{ZC} @ 0.5 \mathrm{~mL} \mathrm{~L}^{-1}$ (0.00) treated plot after seven days of spraying and it was significantly different from the other treatments. The treatment hand mixed product of chlorantraniliprole $18.5 \% \mathrm{SC}+$ thiamethoxam $25 \%$ WG (1:1) recorded a population of 1.00 and it was on par with chlorantraniliprole18.5\% SC @ $0.3 \mathrm{~mL} \mathrm{~L}^{-1}$ (1.33), chlorantraniliprole $8.8 \%+$ thiamethoxam 17.5 \% $\mathrm{SC} @ 0.3 \mathrm{~mL} \mathrm{~L}^{-1}$ (1.33), beta cyfluthrin $8.49 \%$ + imidacloprid $19.81 \% \quad \mathrm{SC} @ 0.4 \quad \mathrm{~mL} \mathrm{~L}^{-1}$ (1.33), flubendiamide19.92\% + thiacloprid $19.92 \%$ SC @ $0.5 \mathrm{~mL} \mathrm{~L}^{-1}$ (1.67). Whereas, number of larvae in thiamethoxam $25 \%$ WG @ $0.3 \mathrm{~mL}$ $\mathrm{L}^{-1}$ and thiamethoxam $12.6 \%$ + lambda cyhalothrin 9.5\% ZC @ $0.3 \mathrm{~mL} \mathrm{~L}^{-1}$ recorded $3.67,2.00$ respectively and they were significantly different when compared with untreated control (6.33).

After ten days of spraying lambda cyhalothrin $4.6 \%$ + chlorantraniliprole $9.3 \%$ ZC @ 0.5 $\mathrm{mL} \mathrm{L}^{-1}$ showed no population of M.vitrata and it was on par with beta cyfluthrin $8.49 \%+$ imidacloprid19.81\% SC @ $0.4 \mathrm{~mL} \mathrm{~L}^{-1}$ (0.67) which was on par with thiamethoxam $12.6 \%$ + lambda cyhalothrin $9.5 \%$ ZC @ $0.3 \mathrm{~mL} \mathrm{~L}^{-1}$ (1.00). The plants treated withchlorantraniliprole $8.8 \%$ + thiamethoxam $17.5 \%$ SC @ $0.3 \mathrm{~mL} \mathrm{~L}^{-1} 0.3 \mathrm{~mL} \mathrm{~L}^{-1}$, hand mixed product of chlorantraniliprole $18.5 \%$ SC +thiamethoxam $25 \%$ WG @ (1:1) showed 1.67 larvae and they were on par with flubendiamide19.92 \% + thiacloprid $19.92 \%$ SC @ $0.5 \quad m L \quad L^{-1} \quad$ (2.00), chlorantraniliprole18.5\% SC @ $0.3 \mathrm{~mL} \mathrm{~L}^{-1}$ (2.00). Thiamethoxam $25 \% \mathrm{WG} @ 0.3 \mathrm{~mL} \mathrm{~L}^{-}$ 1 showed 3.67 larvae which is significantly different from all other treatments including with untreated control (6.33).

No larva was observed in lambda cyhalothrin $4.6 \%$ + chlorantraniliprole $9.3 \%$ ZC @ $0.3 \mathrm{~mL} \mathrm{~L}^{-1}$ after 15 days of spraying and it was significantly different from other treatments. Beta cyfluthrin $8.49 \%+$ imidacloprid $19.81 \%$ SC $@ 0.4 \quad \mathrm{~mL} \mathrm{~L}^{-1}$ showed 1.00 larva and it was on par with treatment hand mixed product of chlorantraniliprole $18.5 \% \mathrm{SC}$ +thiamethoxam $25 \%$ WG (1:1) @ $0.3 \mathrm{~mL} \mathrm{~L}^{-1}$ (1.67), thiamethoxam 12.6\% + lambda cyhalothrin $9.5 \%$ ZC @ $0.3 \mathrm{~mL} \mathrm{~L}^{-1}$ (1.33). More or less similar number of larvae were found in thiamethoxam $25 \%$ WG @ 0.3mL L $\mathrm{mL}^{-1}$ (3.67), chlorantraniliprole18.5\%SC @ $0.3 \mathrm{~mL} \mathrm{~L}^{-1}$ (2.67), flubendiamide19.92 \%+thiacloprid $19.92 \% \quad \mathrm{SC} \quad @ \quad 0.5 \quad \mathrm{~mL} \quad \mathrm{~L}^{-1} \quad$ (2.33), chlorantraniliprole $8.8 \%$ +thiamethoxam 17.5 $\%$ SC @ $0.3 \mathrm{~mL} \mathrm{~L}^{-1}$ (2.00) and they were statistically on par with each other. The highest population was found in untreated control (6.67) (Table 1 and 2). 
Table.1 Insecticide mixtures selected for study

\begin{tabular}{|c|c|c|}
\hline Insecticide mixture & Trade name & Dosage $\left(\mathrm{mL} \mathrm{L}^{-1}\right)$ \\
\hline Chlorantraniliprole $8.8 \%$ + thiamethoxam $17.5 \%$ SC & Voliumflexi & 0.30 \\
\hline $\begin{array}{l}\text { Lambda cyhalothrin } 4.6 \%+\text { chlorantraniliprole } 9.3 \% \\
\text { ZC }\end{array}$ & Ampligo & 0.50 \\
\hline Thiamethoxam $12.6 \%$ + lambda cyhalothrin $9.5 \% \mathrm{ZC}$ & Alika 247 & 0.30 \\
\hline Beta cyfluthrin $8.49 \%+$ imidacloprid $19.81 \%$ SC & Solomon & 0.40 \\
\hline Flubendiamide $19.92 \%$ + thiacloprid $19.92 \% \mathrm{SC}$ & Belt expert & 0.50 \\
\hline $\begin{array}{l}\text { Hand mixing of Chlorantraniliprole } \\
\text { +thiamethoxam } 25 \% \text { WG }(1: 1)\end{array}$ & - & 0.30 \\
\hline Chlorantraniliprole18.5\% SC (check) & Coragen & 0.30 \\
\hline Thiamethoxam $25 \%$ WG (check) & Arrow & 0.40 \\
\hline
\end{tabular}

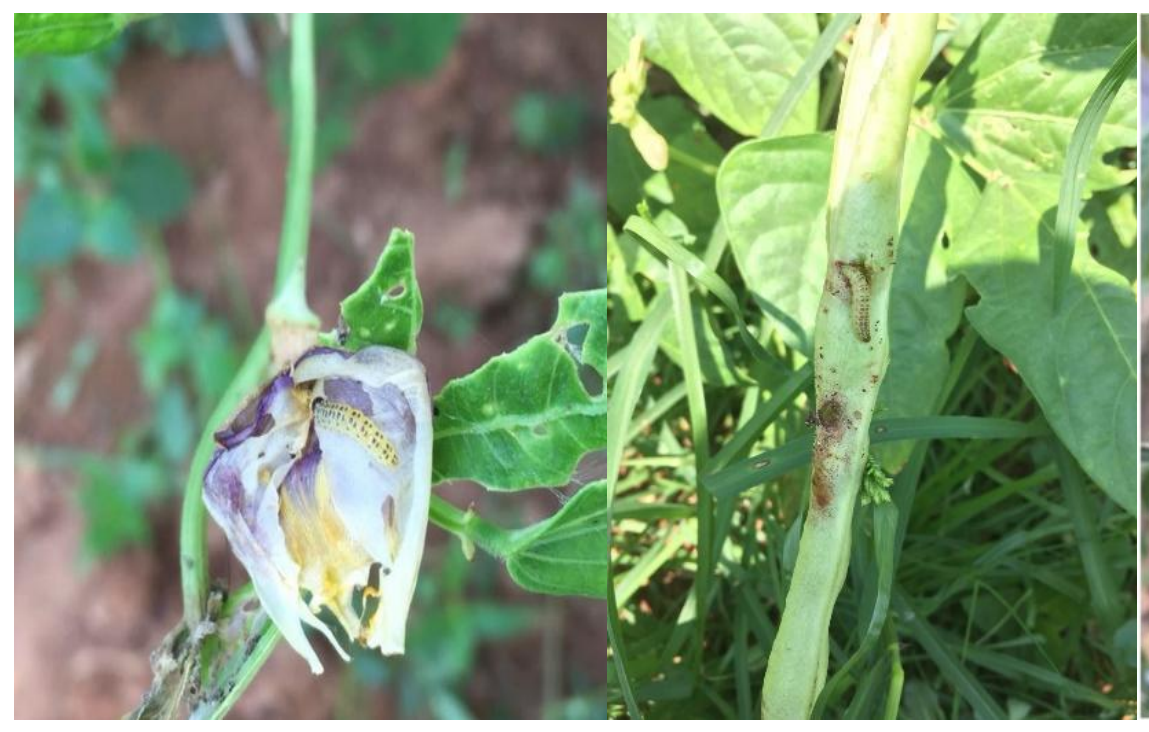

Flower damage
Pod damage

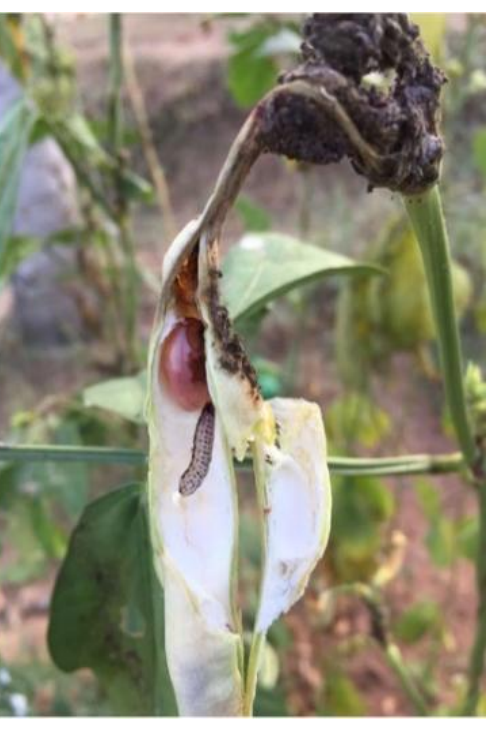

Seed damage 
Table.2 Population of spotted pod borer, Maruca vitrata treated with insecticide mixtures

\begin{tabular}{|c|c|c|c|c|c|c|c|}
\hline \multirow[t]{2}{*}{ Insecticide mixtures } & \multirow{2}{*}{$\begin{array}{c}\text { Field } \\
\text { dose } \\
(\mathrm{mL} \text { or } \mathrm{g} \\
\left.\mathrm{L}^{-1}\right)\end{array}$} & \multicolumn{6}{|c|}{ * Number of larvae per plant (DAS) } \\
\hline & & $\mathbf{1}$ & 3 & 5 & 7 & 10 & 15 \\
\hline $\begin{array}{l}\text { Chlorantraniliprole } 8.8 \quad \% \quad+ \\
\text { thiamethoxam 17.5 \% SC }\end{array}$ & 0.30 & $\begin{array}{c}2.00 \\
(1.41)\end{array}$ & $\begin{array}{c}1.00 \\
(1.22)\end{array}$ & $\begin{array}{c}0 \\
(0.70)\end{array}$ & $\begin{array}{c}1.33 \\
(1.34)\end{array}$ & $\begin{array}{c}1.67 \\
(1.46)\end{array}$ & $\begin{array}{c}2.00 \\
(1.58)\end{array}$ \\
\hline $\begin{array}{l}\text { Lambda cyhalothrin } 4.6 \quad \% \quad+ \\
\text { chlorantraniliprole } 9.3 \% \text { ZC }\end{array}$ & 0.50 & $\begin{array}{c}2.00 \\
(1.41)\end{array}$ & $\begin{array}{c}0.33 \\
(0.87)\end{array}$ & $\begin{array}{c}0 \\
(0.70)\end{array}$ & $\begin{array}{c}0 \\
(0.70)\end{array}$ & $\begin{array}{c}0 \\
(0.70)\end{array}$ & $\begin{array}{c}0 \\
(0.70)\end{array}$ \\
\hline $\begin{array}{l}\text { Thiamethoxam 12.6 \% + lambda } \\
\text { cyhalothrin } 9.5 \% \mathrm{ZC}\end{array}$ & 0.30 & $\begin{array}{c}3.67 \\
(1.91)\end{array}$ & $\begin{array}{c}2.67 \\
(1.77)\end{array}$ & $\begin{array}{c}2.67 \\
(1.77)\end{array}$ & $\begin{array}{c}2.00 \\
(1.55)\end{array}$ & $\begin{array}{r}1.00 \\
(1.22)\end{array}$ & $\begin{array}{c}1.33 \\
(1.34)\end{array}$ \\
\hline $\begin{array}{l}\text { Beta cyfluthrin } 8.49 \%+\text { imidacloprid } \\
19.81 \% \text { SC }\end{array}$ & 0.40 & $\begin{array}{l}2.67 \\
1.62)\end{array}$ & $\begin{array}{c}2.00 \\
(1.58)\end{array}$ & $\begin{array}{c}2.00 \\
(1.58)\end{array}$ & $\begin{array}{c}1.33 \\
(1.34)\end{array}$ & $\begin{array}{c}0.67 \\
(0.99)\end{array}$ & $\begin{array}{c}1.00 \\
(1.17)\end{array}$ \\
\hline $\begin{array}{l}\text { Flubendiamide19.92\% +thiacloprid } \\
19.92 \% \text { SC }\end{array}$ & 0.50 & $\begin{array}{c}3.33 \\
(1.82)\end{array}$ & $\begin{array}{c}1.33 \\
(1.34)\end{array}$ & $\begin{array}{c}0.67 \\
(1.05)\end{array}$ & $\begin{array}{c}1.67 \\
(1.46)\end{array}$ & $\begin{array}{c}2.00 \\
(1.58)\end{array}$ & $\begin{array}{c}2.33 \\
(1.67)\end{array}$ \\
\hline $\begin{array}{l}\text { Hand mixing of Chlorantraniliprole } \\
18.5 \% \text { SC +thiamethoxam } 25 \% \text { WG } \\
(1: 1)\end{array}$ & 0.30 & $\begin{array}{c}1.00 \\
(1.00)\end{array}$ & $\begin{array}{c}0.67 \\
(1.05)\end{array}$ & $\begin{array}{c}1.33 \\
(1.34)\end{array}$ & $\begin{array}{c}1.00 \\
(1.22)\end{array}$ & $\begin{array}{c}1.67 \\
(1.46)\end{array}$ & $\begin{array}{c}1.67 \\
(1.46)\end{array}$ \\
\hline Chlorantraniliprole18.5\% SC (check) & 0.30 & $\begin{array}{c}2.00 \\
(1.41)\end{array}$ & $\begin{array}{c}1.00 \\
(1.17)\end{array}$ & $\begin{array}{c}0.67 \\
(1.05)\end{array}$ & $\begin{array}{c}1.33 \\
(1.34)\end{array}$ & $\begin{array}{c}2.00 \\
(1.57)\end{array}$ & $\begin{array}{c}2.67 \\
(1.77)\end{array}$ \\
\hline Thiamethoxam $25 \%$ WG (check) & 0.40 & $\begin{array}{c}3.67 \\
(1.91)\end{array}$ & $\begin{array}{c}2.67 \\
(1.77)\end{array}$ & $\begin{array}{c}3.33 \\
(1.95)\end{array}$ & $\begin{array}{c}3.67 \\
(2.03)\end{array}$ & $\begin{array}{c}3.67 \\
(2.03)\end{array}$ & $\begin{array}{c}3.67 \\
(2.03)\end{array}$ \\
\hline Control & & $\begin{array}{c}5.67 \\
(2.37)\end{array}$ & $\begin{array}{c}6.33 \\
(2.61)\end{array}$ & $\begin{array}{c}6.67 \\
(2.67)\end{array}$ & $\begin{array}{c}6.33 \\
(2.60)\end{array}$ & $\begin{array}{c}6.33 \\
(2.60)\end{array}$ & $\begin{array}{c}6.67 \\
(2.67)\end{array}$ \\
\hline CD (0.05) & & 0.221 & 0.388 & 0.338 & 0.323 & 0.320 & 0.359 \\
\hline
\end{tabular}

Figures in parentheses are $\sqrt{x}+1$ transformed values, DAS- Days after spraying, *Mean of fifteen plants

\section{Spotted pod borer infestation in cowpea}

By considering results of flower damage, lambda cyhalothrin $4.6 \%+$ chlorantraniliprole $9.3 \% \mathrm{ZC} @ 0.5 \mathrm{~mL} \mathrm{~L}^{-1}$ is the best effective mixture followed by chlorantraniliprole $8.8 \%+$ thiamethoxam $17.5 \% \mathrm{SC} @ 0.3 \mathrm{~mL} \mathrm{~L}^{-1}$.

In 2017, Roy et al., reported similar results in managing pod borer, $M$. vitrata in cowpea by spraying chlorantraniliprole $8.8 \% \quad+$ thiamethoxam 17.5\% SC @ $180 \mathrm{~mL} \mathrm{ha}^{-1}$.

However, lambda cyhalothrin $4.6 \%+$ chlorantraniliprole $9.3 \% \mathrm{ZC} @ 35 \mathrm{~g}$ a.i ha ${ }^{-1}$ was found to be the best in reducing the infestation of borer pests in different crops viz., pigeon pea (Patel and Patel, 2013), soy bean (Birla, 2014), cotton (Bajya et al., 2015), cowpea (Grigolli et al., 2015), brinjal (Sen et al., 2017), pigeon pea (Swami et al., 2017). In Kerala, study conducted by Sreelakshmi et al.,2016 revealed that indoxacarb $14.5 \%+$ acetamiprid 7.7\% SC @ $100 \mathrm{~g}$ a.i ha ${ }^{-1}$ was found to be effective in managing the resistant population of $M$. vitrata and flubendiamide + buprofezin@875 mL ha ${ }^{-1}$ was recorded as best insecticide mixture against borer and sucking pests of rice (Kartikeyan et al., 2012). Several studies has been conducted by using chlorantraniliprole and lambda cyhalothrin as single insecticides against $M$. vitrata. Chlorantraniliprole@0.15 mL L ${ }^{-1}$ was found 
to be superior in reducing larval population of M. vitrata in cowpea (Kumar et al., 2014; Yadav and Singh, 2014), redgram (Kumar et al., 2015), pigeon pea (Jakhar et al., 2016). Toxicity of insecticides against pod borers in pigeon pea showed that lambdacyhalothrin 5 EC @ $25 \mathrm{~g}$ a.i. ha ${ }^{-1}$ was highly effective in reducing pod borer infestation in pigeon pea (Mohapatra and Srivastava, 2002; Koushik and Pal, 2006; Dhaka et al., 2011; Priyadarshini et al., 2013), Indian bean (Viroja, 2003), green gram (Rani and Eswari, 2008) and in black gram (Sonune et al., 2010).

\section{References}

Bajya, D.R.,Baheti, H.S., and Raza, S. K.2015. Field efficacy of newer insecticide formulation Ampligo $150 \mathrm{ZC}$ against bollworm complex in cotton. J. Cotton. Res. Dev. 29 (1): 94-98.

Birla,D. 2014. Comparative field efficacy of combination insecticide against insect pests of soybean, Glycine max (L.) Merr. M. Sc. thesis, Rajamata Vijayaraje Scindia Krishi Vishwa Vidyalaya, Indore, 93.p.

Dhaka, S.S., Singh, G., Yadav, A., Rai, M., and Kumar, A. 2011. Efficacy of novel insecticides against pod borer, Helicoverpa armigera (Hubner) in Pigeon pea. Prog. Hortic.47(1):98-102.

Grigolli, J.F.J., Luis, A., Lourencao, F., and Avila, C. J. 2015. Field Efficacy of Chemical Pesticides against Maruca vitrata Fabricius (Lepidoptera: Crambidae) Infesting Soybean in Brazil. American J Plant Sci. 6: 537-544.

Halder, J., and Srinivasan, S. 2011. Varietal screening and role of morphological factors on distribution and abundance on spotted pod borer, Maruca vitrata (Fabricius) on cowpea. Ann. Pl. Protec. Sci. 19: 71-74.

IRAC [Insecticide Resistance Action committee] 2018. IRAC home page [online]. Available: http://www.irac- online.org

Jakhar, B. L., Kumar, S., and Ravindrababu, Y. 2016. Efficacy of different newer insecticides against legume pod borer, Maruca vitrata (Geyer) on pigeonpea. Res. Crops. 17(1): 134-136.

Kartikeyan, K., Purushothaman, S. M., Smitha, S. G., and Ajish, P. G. 2012. Efficacy of a New Insecticide Combination Against Major Pests of Paddy. Indian J. Plant Prot.40(4): 276-279.

Kaushik, D., and Pal, D. P. B 2006. Bioefficacy of different insecticides against pod borer complex in pigeon pea. Environ. Ecol. 24: 184-186.

Kumar, S., Durairaj, C., Ganapathy, N., and Kumar, M. S. 2015. Field evaluation of newer insecticide molecules and botanicals against pod borers of Red gram. Legume Res.38(2): 260-267.

Kumar, S., Pal, S., Lal, G., Singh, D. K., and Umrao, R. S. 2014. Bio-efficacy of insecticides and bio-pesticides against pod borer and jassids on Cowpea, Vigna uniguiculata (L.) Ann. Pl. Protec. Sci. 22(2): 264-267.

Mallet, J. 1989. The evolution of insecticide resistance: have the insects won. Tree. 4: 336-340.

Mani, G.S. 1985. Evolution of resistance in the presence of two insecticides. Genetics. 109:761-783.

Mohapatra, S. D., and Srivastava, C. P. 2002. Bio-efficacy of chemical and biorational insecticides against incidence of legume pod borer, Maruca vitrata (Geyer) in short duration pigeonpea. Indian $J$. Plant Prot. 30 (1): 22-25.

Pandey, S.N., Singh, R., Sharma, V.K., and Kanwat, P.W. 1991. Losses due to insect pests in some Kharif Pulses. Indian J. Ent. 53 (4): 629-631.

Patel, S.A., and Patel, R.K. 2013. Bio-efficacy of newer insecticides against pod borer complex of pigeonpea [Cajanus cajan (L.) millspaugh]. AGRES - An International e-Journal. 2 (3): 398-404.

Priyadarshini, G., Reddy, C. N., and Reddy, D. J. 2013. Bio-efficacy of selective 
insecticides against lepidopteran pod borers in pigeonpea. Indian J. Plant Prot. 41(1): 6-10.

Rani, C. S. and Eswari, K. B. 2008. Evaluation of some newer insecticides against maruga on green gram. Asian $J$. Biosci.3(2): 346-347.

Roy, D., Chakraborty, G., and Sarkar, P. K. 2017. Comparative efficacy, non-target toxicity and economics of seven novel premixed formulations against Maruca testulalis G. and Aphis craccivora K. infesting cowpea. J. Environ. Biol.38: 603-609.

Sardhana, H.R., and Verma,S. 1986. Preliminary studies on the prevalence of insect pests and their natural enemies on cowpea crop in relation to weather factors at Delhi. Indian. J. Ent.48(4): 242-244.

Sen, K., Samanta, A., Alam, S. K. F., and Dhar, P. P. 2017. Field evaluation of a New Ready-Mix Formulation Lambda cyhalothrin $4.6 \%$ + chlorantraniliprole $9.3 \%$ ZC) against Shoot and Fruit Borer (Leucinodes orbonalis Guen.) infestation in Brinjal. J.Pharmacogn.Phytochem. 6(5): 16741678.

Skylakakis, G. 1981. Effects of alternating and mixing pesticides on the build-up of fungal resistance. Phytopathol. 71: 1119-1121.
Sonune, V. R., Bharodia, R. K., Jethva, D. M., Rathod, R. T., and Deshmukh, S. G. 2010. Field efficacy of chemical insecticides against spotted pod borer, Maruca vitrata (F.) infesting blackgram. Legume Res.33(4): 287-290.

Sreelakshmi, P., Paul, A., Beevi, N. S., Sheela, M. S., and Kumar, P. N. 2016. Management of Resistant Populations of Legume Pod Borer, Maruca vitrata (Fab.) (Lepidoptera: Crambidae) using new generation insecticides. Environ. Ecol. 34(3): 917-921Swami, Hemant, Ameta OP and Lekha (2017) Bioefficacy of novel insecticides against pod borer, (Helicoverpa armigera Hubner) in pigeonpea. Legume Res.40(4): 756-761.

Vijayasree, V. 2013. Efficacy and Biosafety of new generation insecticides for the management of fruit borers of cowpea. Ph.D. thesis. Kerala Agricultural University. 220.p.

Viroja, K. J. 2003. Efficacy of conventional insecticides for management of Pod borers in Indian bean. M. Sc. thesis, Junagadh Agricultural University, Junagadh, $159 \mathrm{p}$.

Yadav, N. K., and Singh, P. S. 2014. Bioefficacy of chemical Insecticides against Spotted Pod Borer, Maruca testulalis (Geyer) on Cowpea. IJAEB: 7(1): 187190.

\section{How to cite this article:}

Banka Kanda Kishore Reddy and Jangam Hampaiah. 2018. Evaluation of Insecticide Mixtures against Larval Population of Spotted Pod Borer, Maruca vitrata in Cowpea. Int.J.Curr.Microbiol.App.Sci. 7(07): 1820-1826. doi: https://doi.org/10.20546/ijcmas.2018.707.215 